\title{
Contrast Harmonic Endoscopic Ultrasound in Pancreatic Diseases
}

\author{
Rafael Mejuto-Fernandez and Julio Iglesias-Garcia \\ Department of Gastroenterology and Hepatology, Health Research Institute (IDIS), University Hospital of Santiago de Compostela, \\ Santiago de Compostela, Spain
}

\begin{abstract}
Endoscopic ultrasound (EUS) was first described in 1986, with the aim of overcoming the problems affecting transabdominal ultrasound imaging, mainly problems related to the interposition of gas, and artifacts produced by bone or fat. Now, EUS can be considered as the best method for the analysis of pancreatic diseases, overtaking the diagnostic accuracy of computed tomography and magnetic resonance imaging. However, fundamental B-mode imaging is limited for the diagnosis of solid pancreatic lesions, because most of them are depicted as heterogeneous and hypo-echoic, and it is difficult to differentiate between benign and malignant lesions. Similar to how perfusion patterns obtained by computed tomography or magnetic resonance imaging after injection of contrast agents allow for the characterization of focal lesions, EUS has also recently been introduced to the use of contrast agents for performing contrast-enhanced harmonic EUS (CEH-EUS), which has the capability to distinguish the type of perfusion between lesions and surrounding tissue. CEH-EUS has shown its usefulness for the diagnosis and characterization of solid pancreatic lesions. Moreover, CEH-EUS is also highly accurate for distinguishing non-neoplastic from neoplastic cysts in pancreatic lesions. Another potential role of CEH-EUS is its ability to direct EUS-guided tissue acquisition. Clin Endosc 2021;54:309-313
\end{abstract}

Key Words: Contrast enhancement; Endoscopic ultrasound; Pancreatic tumors

\section{INTRODUCTION}

Endoscopic ultrasound (EUS) was introduced in the mid 1980s to avoid problems affecting transabdominal ultrasound imaging, such as the interposition of gas and the artifacts obtained from bone and fat. EUS is now considered a diagnostic and therapeutic method for the management of gastrointestinal and extraintestinal diseases. EUS has evolved, becoming the method of choice for the evaluation of the pancreaticobiliary system such as for pancreatic lesions (both solid and

Received: February 17, 2020 Revised: April 9, 2020

Accepted: April 9, 2020

Correspondence: Julio Iglesias-Garcia

Department of Gastroenterology and Hepatology, Health Research Institute (IDIS), University Hospital of Santiago de Compostela, c/Choupana s/n, Santiago de Compostela 15706, Spain

Tel: +34-981-951-364, Fax: +34-981-955-100, E-mail: julioiglesiasgarcia@gmail. com

ORCID: https://orcid.org/0000-0002-3517-329X

It is the invited review article.

cc This is an Open Access article distributed under the terms of the Creative Commons Attribution Non-Commercial License (http://creativecommons.org/ licenses/by-nc/3.0) which permits unrestricted non-commercial use, distribution, and reproduction in any medium, provided the original work is properly cited. cystic), showing superiority over other imaging techniques, such as computed tomography (CT) and magnetic resonance imaging (MRI). However, fundamental B-mode EUS imaging has certain limitations, mainly in the differential diagnosis of pancreatic tumors and for the evaluation of patients with suspected chronic pancreatitis. In order to overcome these drawbacks, new EUS systems have been designed, enabling the use of high-frequency scanning systems and different associated technologies. The main ones among them are Doppler evaluation, real-time elastography, and contrast enhancement. Recent publications have demonstrated the technique and diagnostic capabilities of contrast enhanced harmonic EUS (CEH-EUS). ${ }^{1}$

In this article, we will summarize the current knowledge of $\mathrm{CEH}-\mathrm{EUS}$ in pancreatic diseases.

\section{HISTORY OF CONTRAST ENHANCED ENDOSCOPIC ULTRASOUND}

Contrast enhanced EUS was initially reported in the 1990s with the use of intra-arterial infusion of $\mathrm{CO}_{2}$. However, this 
method could only be performed during angiographic examinations. The next advancement was the possibility of performing color and power Doppler sonography under EUS guidance (CD-EUS). In the mid 1990s, the development of contrast agents deliverable by vein access provided the opportunity to obtain images without angiographic support. Combining CD-EUS with contrast agents enabled the opportunity to generate pseudo-Doppler signals from microbubbles that increased imaging capabilities. ${ }^{2}$ Nevertheless, CD-EUS is hampered by different artifacts including motion artifacts, blooming, and overpainting. In order to decrease these artifacts, visualization of micro-vessels and parenchymal perfusion can be obtained by CEH-EUS with second-generation contrast agents and broadband EUS transducers developed at the beginning of the 21 st century. ${ }^{3}$

\section{CONTRAST AGENTS, HOW AND WHY THE WORK}

Currently, contrast agents are formed using microbubbles of $2-5 \mu \mathrm{m}$ in diameter, which are allowed to go through the lung in order to induce a systemic enhancement after intravenous injection. These microbubbles have a resistant shell that allows enhancement before the pressure leads to dissolution or disruption. They are also filled with a heavy gas (like perfluorocarbon), which has a lower probability of leaking out of the bubbles and remains in circulation longer, making assessment of tissue perfusion easier.

Three generations of contrast agents have been developed. The first-generation agents consisted in microbubbles filled with air, requiring high acoustics to obtain adequate imaging. Second-generation agents included Definity (octafluoropropane), Sonazoid (perfluorobutane), and SonoVue (sulfur-hexafluoride). At low acoustic power, second-generation agents produce harmonic signals, considered ideal for EUS, mainly due to the small size of the transducer. Among these agents, Sonazoid has the capability to present a late hepato-splenic phase, because microbubbles can be trapped in Kupffer cells. The development of third-generation of contrast agents is on-going. EchoGen ${ }^{\circledR}$ (dodecafluoropentane; Sonus Pharmaceuticals Ltd., Bothell, WA, USA) is capable of shifting from lipid to gas when reaching body temperature, providing better imaging; however, these types of agents have not been used in EUS, and their role is currently limited to the evaluation of cardiac diseases. ${ }^{2}$

The effect of contrast agents is based on the greater compressibility of the gases, whereas tissue is relatively incom- pressible. When an ultrasound wave hits a bubble of gas, this wave is reflected at the interface between the bubble and the surrounding tissue, occasioned by the difference in acoustic impedance. Microbubbles undergo a volumetric oscillation, and vibration of these microbubbles induces an acoustic signal, which can be detected and reproduced. ${ }^{4}$

Contrast agents are considered to be safe with a low number of side effects. Contrast agents can be safely administered in patients suffering from renal insufficiency. In fact, there is no need for a laboratory test before the study. The reported incidence of allergic reaction is even lower than that reported for standard contrast agents used in radiological explorations. Conditions in which caution should be taken include severe coronary disease and severe pulmonary hypertension. ${ }^{5}$

\section{HOW TO PERFORM A CONTRAST ENHANCED HARMONIC ENDOSCOPIC ULTRASOUND}

The extended pure harmonic mode is needed for CEHEUS. Harmonic imaging produces images based on a non-linear acoustic effect of the interaction of ultrasound with tissues or contrast agents. ${ }^{3}$ The system combines the reception of the filtered fundamental and second harmonic frequency components. In fact, circulation can be better evaluated by specifically detecting the harmonic signal components from the microbubbles inside the vessels. For an optimal image, a low mechanical index (between 0.08 and 0.25 ) with its corresponding power is needed (dynamic wide-band contrast harmonic imaging mode).

Certain steps are needed to perform CEH-EUS. For the analysis, a two-panel image is shown with the conventional gray-scale fundamental B-mode at one side of the screen and the contrast harmonic image on the other side. Once the system has been set up, the contrast agent needs to be slowly injected directly into the access vein through a catheter of 16 or 18 gauge in order to avoid the destruction of the bubbles inside the system. ${ }^{6}$ After injecting the contrast agent, the catheter must be flushed with saline to clear out persistent microbubbles. Ideally, $10 \mathrm{~mL}$ of saline may be administered in order to optimize the study. Two phases of contrast enhancement can be analyzed after injecting the contrast agent: (1) an early/ arterial phase (from 10-30 seconds), starting when a hyperechogenic appearance of the aorta or other major arteries are observed, and (2) a venous/late phase (from 30-120 seconds), defined by the presence an hyperechogenicity of the splenic and mesenteric vessels. ${ }^{3}$ 


\section{MAIN PANCREATIC INDICATIONS FOR CONTRAST ENHANCED HARMONIC ENDOSCOPIC ULTRASOUND}

CEH-EUS has shown a key role in two of the main pancreatic indications: solid and cystic pancreatic lesions.

\section{Solid pancreatic lesions}

Differential diagnosis of solid pancreatic tumors is very challenging, based on current imaging techniques, mainly due to the difficulty of differentiating pancreatic adenocarcinomas from other type of pancreatic tumors.

The capability of EUS to distinguish benign from malignant lesions is limited, despite recent technical developments associated with image quality and resolution. In this context, EUS-guided tissue acquisition has clearly improved the diagnostic accuracy of EUS; however, there are certain drawbacks that need to be highlighted. Among them are the risk of false-negative results and the small, but not negligible, rate of potential complications. Furthermore, EUS-guided tissue ac- quisition can be technically demanding and difficult in certain cases, mostly associated with problems in obtaining the correct position of the echoendoscope or the needle, and to the presence of interposed tissue or vascular structures. Considering these facts, the development of an accurate and minimally invasive technology leading to an optimized EUS diagnosis seems to be essential for optimizing the management of these cases.

When evaluating solid pancreatic tumors, there are well-described contrast enhancement patterns. Pancreatic adenocarcinoma mostly appears as a hypovascular lesion (Fig. 1), whereas most of the inflammatory lesions (such as mass-forming chronic pancreatitis or autoimmune pancreatitis) present an isovascular pattern. On the other hand, a hypervascular pattern is mainly present in neuroendocrine tumors and some pancreatitis metastases (like metastasis from renal cancer) (Fig. 2). According to several studies, the presence of a hypovascular or even a hypervascular pattern on CEH-EUS is associated with pancreatic malignancy with a sensitivity of $89.8 \%$ and a specificity of $69.2 \%{ }^{6}$
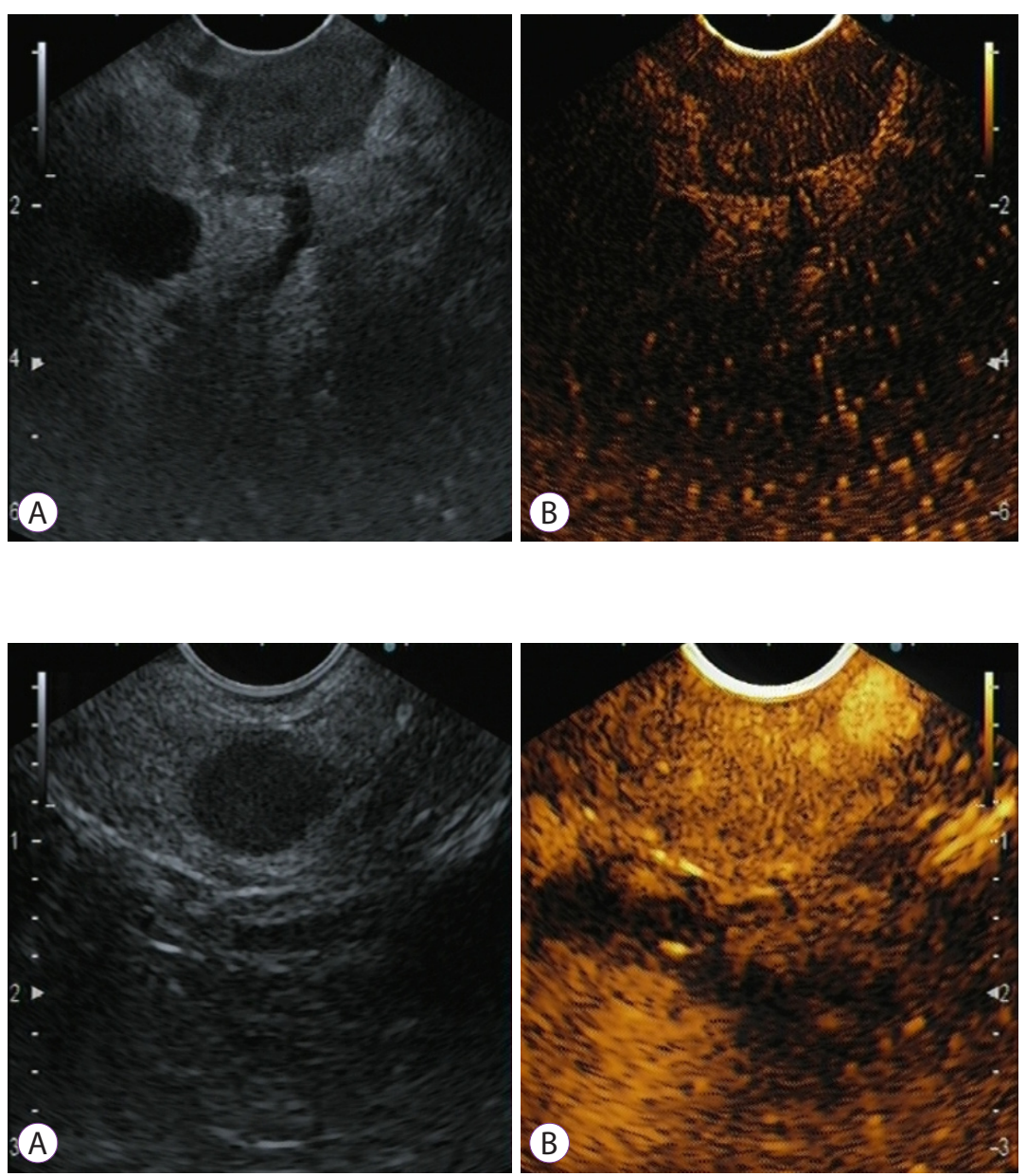

Fig. 2. Contrast-enhanced harmonic endoscopic ultrasound (CEH-EUS) evaluation of pancreatic solid mass, at the end of the arterial phase and beginning of the venous phase (00:40 minutes). The pancreatic lesion is detected as a low-echoic lesion with fundamental B-mode EUS (A). CEH-EUS detects a pancreatic lesion with hyperenhancement in comparison with the surrounding pancreatic tissue (B). 

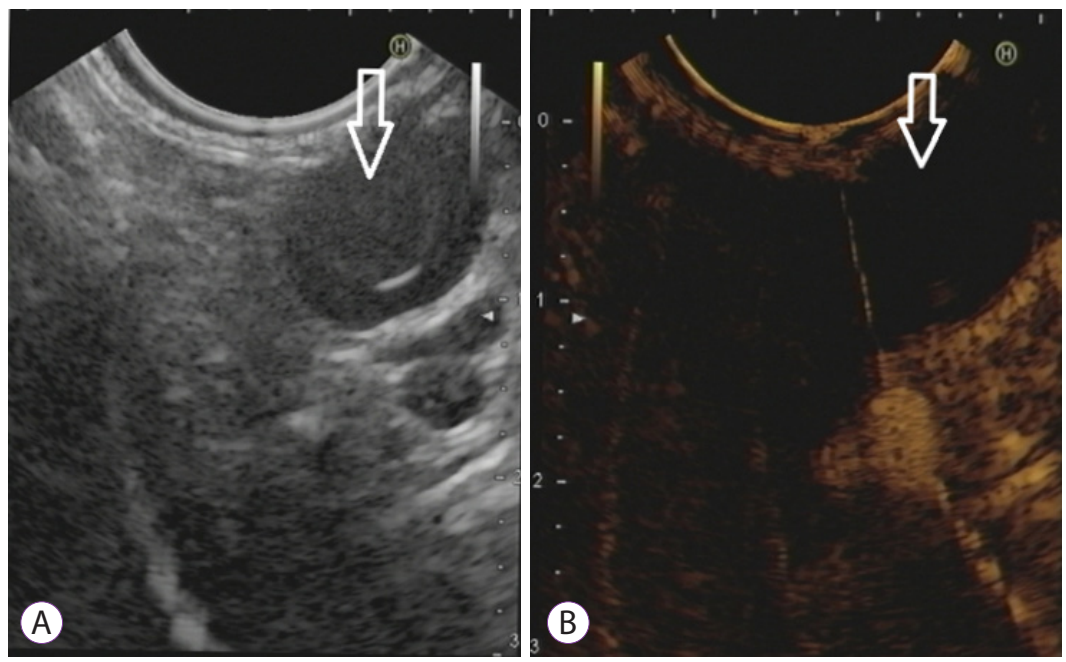

Fig. 3. Contrast-enhanced harmonic endoscopic ultrasound (CEH-EUS) for an intraductal papillary mucinous neoplasm with a suspicion of mural lesion: a representative mucous clot case. Fundamental B-mode EUS (A) shows a hyperechoic lesion (arrow) in a cyst cavity. CEH-EUS (B) detects no vascularity in the lesion (arrow).

\section{Cystic pancreatic lesions}

Pancreatic cystic lesions (PCL) are a frequent incidental finding. CEH-EUS allows for distinguishing between pseudocysts and PCL, based on the demonstration of the vascularization of the septa of the lesion and the nodules. Among pancreatic cystic tumors, mucinous cystic tumors and intraductal papillary mucinous neoplasms (IPMNs) have a malignant potential. However, the decision to perform a surgical treatment must be carefully made since IPMNs usually grow slowly, present a variety of histological subtypes with different malignant potential, and are usually detected in older patients. Therefore, after detecting PCL, a complete workup is needed to plan the best management. In this context, it is crucial to correctly characterize a lesion as benign, premalignant, or malignant. At this point CEH-EUS appears as a very useful tool, helping in the differential diagnosis of PCL, with the capability to identify high-risk stigmata and/or worrisome features, by enabling the assessment of the vascularization of different structures such as cyst walls, septa, or mural nodules. Discrimination of contrast-enhancing mural nodules from non-enhancing mucin plugs has become one of the main indications of CEH-EUS in the evaluation of PCL (Fig. 3).

Harima et al. ${ }^{7}$ reported a diagnostic accuracy for mural nodules of $92 \%$ for CT, $72 \%$ for EUS, and $98 \%$ for CEHEUS. CEH-EUS was able to detect more mural nodules when compared to CT $(p<0.05)$ or EUS $(p<0.01)$. Kamata et al. ${ }^{8}$ compared CEH-EUS and fundamental B-mode EUS for the differential diagnosis of PCL, depending on the presence of mural nodules. They found that the $75 \%$ specificity of CEHEUS was clearly more accurate than the $40 \%$ specificity of fundamental B-mode EUS imaging. Fujita et al. ${ }^{9}$ observed that CT, MRI, and EUS were able to detect mural nodules in $86 \%$,
$71 \%$, and $100 \%$ of cases, respectively. However, EUS fundamental B-mode imaging was not able to differentiate mucin plugs from real mural nodules. Importantly, based on CEHEUS, authors could correctly classify all cases based on the vascular pattern. CEH-EUS allowed for differentiation between pseudocysts and other PCL but could not distinguish between mucinous and serous cysts. Mural nodules inside PCL were clearly shown by CEH-EUS as solid components with features of hyperenhancement; this helped to avoid the issue of mucus clots. $^{10}$

\section{CONCLUSIONS}

EUS is superior to other conventional techniques (CT, MRI) for the diagnosis and characterization of pancreatic diseases due to its high spatial resolution. However, one of its remaining limitations is the differential diagnosis between certain types of pancreatic lesions, mainly when they are detected as hypoechogenic nodules. The development of CEH-EUS has led to an improvement in this context, increasing the accuracy of EUS for both the detection and characterization of solid pancreatic lesions. On the other hand, CEH-EUS has shown an excellent ability for the evaluation of PCL, mainly in the detection of mural nodules in the context of a mucinous lesion.

\footnotetext{
Conflicts of Interest

The authors have no potential conflicts of interest.

Funding

None.
} 
ORCID

Rafael Mejuto-Fernandez:

https://orcid.org/0000-0002-9894-6755

Julio Iglesias-Garcia:

\section{REFERENCES}

1. Iglesias-Garcia J, Lariño-Noia J, Domínguez-Muñoz JE. Contrast harmonic endoscopic ultrasound: instrumentation, echoprocessors, and echoendoscopes. Endosc Ultrasound 2017;6:37-42.

2. Kitano M, Yamashita Y. New imaging techniques for endoscopic ultrasonography: contrast-enhanced endoscopic ultrasonography. Gastrointest Endosc Clin N Am 2017;27:569-583.

3. Alvarez-Sánchez MV, Napoléon B. New horizons in the endoscopic ultrasonography-based diagnosis of pancreatic cystic lesions. World J Gastroenterol 2018;24:2853-2866.

4. Sanchez MV, Varadarajulu S, Napoleon B. EUS contrast agents: what is available, how do they work, and are they effective? Gastrointest Endosc 2009;69(2 Suppl):S71-S77.

5. Sidhu PS, Cantisani V, Dietrich CF, et al. The EFSUMB guidelines and

recommendations for the clinical practice of contrast-enhanced ultrasound (CEUS) in non-hepatic applications: update 2017 (long version). Ultraschall Med 2018;39:e2-e44.

6. Iglesias-Garcia J, Lindkvist B, Lariño-Noia J, Abdulkader-Nallib I, Dominguez-Muñoz JE. Differential diagnosis of solid pancreatic masses: contrast-enhanced harmonic (CEH-EUS), quantitative-elastography (QE-EUS), or both? United European Gastroenterol J 2017;5:236-246.

7. Harima H, Kaino S, Shinoda S, Kawano M, Suenaga S, Sakaida I. Differential diagnosis of benign and malignant branch duct intraductal papillary mucinous neoplasm using contrast-enhanced endoscopic ultrasonography. World J Gastroenterol 2015;21:6252-6260.

8. Kamata K, Kitano M, Omoto S, et al. Contrast-enhanced harmonic endoscopic ultrasonography for differential diagnosis of pancreatic cysts. Endoscopy 2016;48:35-41.

9. Fujita M, Itoi T, Ikeuchi N, et al. Effectiveness of contrast-enhanced endoscopic ultrasound for detecting mural nodules in intraductal papillary mucinous neoplasm of the pancreas and for making therapeutic decisions. Endosc Ultrasound 2016;5:377-383.

10. Fusaroli P, Serrani M, De Giorgio R, et al. Contrast harmonic-endoscopic ultrasound is useful to identify neoplastic features of pancreatic cysts (with videos). Pancreas 2016;45:265-268. 